\title{
Continuous Positive Airway Pressure Treatment of Sleepy Patients with Milder Obstructive Sleep Apnea Results of the CPAP Apnea Trial North American Program (CATNAP) Randomized Clinical Trial
}

\author{
Terri E. Weaver 1,2,3, Cristina Mancini ${ }^{2}$, Greg Maislin ${ }^{3,4}$, Jacqueline Cater ${ }^{3,4}$, Bethany Staley ${ }^{3}$, \\ J. Richard Landis ${ }^{5}$, Kathleen A. Ferguson ${ }^{6}$, Charles F. P. George ${ }^{6}$, David A. Schulman7, \\ Harly Greenberg ${ }^{8}$, David M. Rapoport ${ }^{9}$, Joyce A. Walsleben ${ }^{9}$, Teofilo Lee-Chiong ${ }^{10}$, \\ Indira Gurubhagavatula ${ }^{3}$, and Samuel T. Kuna ${ }^{3,11}$
}

\begin{abstract}
${ }^{1}$ Department of Biobehavioral Health Science, University of Illinois at Chicago College of Nursing, Chicago, Illinois; ${ }^{2}$ Biobehavioral and Health Sciences Division, University of Pennsylvania School of Nursing, Philadelphia, Pennsylvania; ${ }^{3}$ Center for Sleep and Circadian Neurobiology, Division of Sleep Medicine, Department of Medicine, and ${ }^{5}$ Center for Clinical Epidemiology and Biostatistics, University of Pennsylvania School of Medicine, Philadelphia, Pennsylvania; ${ }^{4}$ Biostatistical Consulting, Wynnewood, Pennsylvania; ${ }^{6}$ Division of Respirology, Schulich School of Medicine and Dentistry, University of Western Ontario, London, Ontario, Canada; ${ }^{7}$ Division of Pulmonary, Allergy and Critical Care Medicine, Emory University, Emory University Hospital, Atlanta, Georgia; ${ }^{8}$ Division of Pulmonary, Critical Care and Sleep Medicine, North Shore-Long Island Jewish Health System, New Hyde Park, New York; ${ }^{9}$ Division of Pulmonary and Critical Care and Sleep Medicine, Department of Medicine, New York University School of Medicine, New York University Sleep Disorder Center, New York, New York; ${ }^{10}$ Department of Medicine, National Jewish Health, Denver, Colorado; and ${ }^{11}$ Philadelphia Veterans Affairs Medical Center, Philadelphia, Pennsylvania
\end{abstract}

Rationale: Twenty-eight percent of people with mild to moderate obstructive sleep apnea experience daytime sleepiness, which interferes with daily functioning. It remains unclear whether treatment with continuous positive airway pressure improves daytime function in these patients.

Objectives: To evaluate the efficacy of continuous positive airway pressure treatment to improve functional status in sleepy patients with mild and moderate obstructive sleep apnea.

Methods: Patients with self-reported daytime sleepiness (Epworth Sleepiness Scale score $>10$ ) and an apnea-hypopnea index with $3 \%$ desaturation and from 5 to 30 events per hour were randomized to 8 weeks of active or sham continuous positive airway pressure treatment. After the 8-week intervention, participants in the sham arm received 8 weeks of active continuous positive airway pressure treatment.

Measurements and Main Results: The Total score on the Functional Outcomes of Sleep Questionnaire was the primary outcome measure.

(Received in original form February 5, 2012; accepted in final form July 5, 2012)

Supported by National Institutes for Health National Heart, Lung, and Blood Institute, R01 HL076101 (T.E.W., C.M., B.S., J.R.L., K.A.F., C.F.P.G., D.A.S., H.G., D.M.R., J.A.W., S.T.K.); Sleep Medicine Education and Research Foundation (American Academy of Sleep Medicine) (T.E.W.); Respironics Sleep and Respiratory Research Foundation (T.E.W.); and Cephalon, Inc. (T.E.W., C.M.). Equipment provided by Philips Respironics, Inc., ProTech Services, Inc., and Embla.

Author Contributions: T.E.W. was responsible for the study design, conduct of the study, data collection and interpretation, and writing of the manuscript. C.M. served as Project Manager, and was responsible for execution of study procedures, study quality control, and data collection. G.M. was the blinded biostatistician on the study, collaborated on the study, conducted the primary data analysis, provided interpretation of the results, and contributed to the writing of the manuscript. J.C. served as the unblinded biostatistician on the study and conducted required data analysis for the DSMB before unblinding. B.S. was responsible for study design and data collection and interpretation. J.R.L. contributed to study design and served for a period of time as Executive Secretary of the Data Safety Monitoring Board. K.A.F., C.F.P.G., D.A.S., H.G., D.M.R., J.A.W., and T.L.-C. contributed to study design, supervised data collection, interpreted the data, and edited and reviewed the manuscript. I.G. provided interpretation and quality control for the blood pressure obtained from the 48-hour ambulatory monitoring data. S.T.K. was responsible for study design, data collection, interpretation of the data, and writing of the manuscript.

Correspondence and requests for reprints should be addressed to Terri E. Weaver, Ph.D., R.N., University of Illinois at Chicago College of Nursing, 845 South Damen Avenue (MC 802), Chicago, IL 60612. E-mail: teweaver@uic.edu

This article has an online supplement, which is accessible from this issue's table of contents at www.atsjournals.org

Am J Respir Crit Care Med Vol 186, Iss. 7, pp 677-683, Oct 1, 2012

Copyright $\odot 2012$ by the American Thoracic Society

Originally Published in Press as DOI: 10.1164/rccm.201202-02000C on July 26, 2012

Internet address: www.atsjournals.org

\section{AT A GLANCE COMMENTARY}

Scientific Knowledge on the Subject

One in five men suffers from mild to moderate obstructive sleep apnea (OSA), 28\% of whom experience excessive daytime sleepiness. Continuous positive airway pressure (CPAP) is the primary treatment for OSA, but this efficacy has been demonstrated in those with more severe disease. It remains unclear whether CPAP is effective in the largest segment of the OSA population, particularly with respect to daily functioning and daytime sleepiness. The few randomized controlled trials of CPAP efficacy in patients with milder OSA have produced conflicting results, principally because of methodologic limitations.

What This Study Adds to the Field

Sleepy patients with mild and moderately severe OSA had greater functional improvement after 8 weeks of CPAP therapy compared with sham CPAP. Compared with placebo, CPAP treatment also produced clinically meaningful changes in mood and self-reported daytime sleepiness. As a multisite study conducted at large and smaller clinical practice sites, our results indicate the efficacy of this therapy in treating sleepy patients with less severe OSA.

The adjusted mean change in the Total score after the first 8-week intervention was 0.89 for the active group $(n=113)$ and -0.06 for the placebo group $(n=110)(P=0.006)$. The group difference in mean change corresponded to an effect size of 0.41 ( $95 \%$ confidence interval, 0.14-0.67). The mean (SD) improvement in Functional Outcomes of Sleep Questionnaire Total score from the beginning to the end of the crossover phase $(n=91)$ was $1.73 \pm 2.50(t[90]=6.59 ; P<$ 0.00001 ) with an effect size of 0.69 .

Conclusions: Continuous positive airway pressure treatment improves the functional outcome of sleepy patients with mild and moderate obstructive sleep apnea.

Clinical trial registered with www.clinicaltrials.gov (NCT 00127348).

Keywords: continuous positive airway pressure; obstructive sleep apnea; daytime sleepiness; randomized clinical trial; functional status 
Obstructive sleep apnea (OSA) is characterized by episodic collapse of the pharyngeal airway during sleep causing intermittent hypoxemia and fragmented sleep. OSA is common; up to $28 \%$ of females and $26 \%$ of males have five or more apneas and hypopneas per hour of sleep (apnea-hypopnea index [AHI]) with $28 \%$ of this population reporting excessive daytime sleepiness $(1,2)$. Based on the AHI, disease severity is categorized as mild $(5 \leqslant$ AHI $<15$ events $/ \mathrm{h})$, moderate (AHI $15-30$ events/h), and severe (AHI > 30 events/h) (3). OSA is associated with premature death, hypertension, ischemic heart disease, stroke, insulin resistance, and work- and driving-related accidents (4-7).

Continuous positive airway pressure (CPAP) is the primary treatment for OSA. CPAP prevents pharyngeal airway collapse during sleep, thereby improving the quality of sleep and oxygen saturation (8). CPAP is reported to improve daytime sleepiness and other daytime impairments, reduce cardiovascular risk, improve insulin sensitivity, increase neurobehavioral performance, and enhance quality of life (9-11). However, this evidence is based mostly on studies of patients with severe OSA (12). It remains unclear whether CPAP is effective in those with milder disease, particularly with respect to daily functioning and daytime sleepiness. The few randomized controlled trials (RCT) of CPAP efficacy in patients with milder OSA have produced conflicting results, principally because of methodologic limitations (9). The purpose of the CPAP Apnea Trial North American Program (CATNAP) was to determine the efficacy of CPAP treatment for functional improvement in sleepy patients with mild and moderate OSA. In this double-blind, randomized, placebo-controlled, parallel-groups study, we hypothesized that the mean change in functional status after 8 weeks of treatment would be greater in participants receiving active CPAP compared with sham CPAP, the placebo intervention. We also hypothesized an improvement in the change in secondary outcomes (mean self-reported sleepiness, objectively measured sleepiness, mood, and mean arterial blood pressure) at 8 weeks posttreatment in those individuals treated with active CPAP compared with sham CPAP.

\section{METHODS}

\section{Sample}

Participants were recruited from consecutive patients. Eligibility criteria included patients with newly diagnosed milder OSA (AHI 5-30 events/ h) who were naive to CPAP and had an Epworth Sleepiness Scale (ESS) score greater than 10 (13). Additionally, participants had a stable medical condition in the past 3 months; greater than fifth grade reading level; and no history of other sleep disorder, current pregnancy, substance abuse, sleepiness-related driving accident, or sleepinesssensitive occupation. The study was approved by the Institutional Review Board at each participating site and informed consent was obtained from all participants.

The primary endpoint was the change after 8 weeks of treatment in Functional Outcomes of Sleep Questionnaire (FOSQ) Total score (see online supplement) (14). Secondary analyses included the FOSQ subscale scores; generic functional status (SF-36) (15); self-reported sleepiness (ESS score) (13); objective sleepiness (lapses in attention measured by the Psychomotor Vigilance Task [PVT]) (16); mood (Total Mood Disturbance scale on the Profile of Mood States [POMS]) (17); and mean 48-hour ambulatory blood pressure (see online supplement).

\section{Procedures}

Diagnostic and CPAP titration polysomnograms (PSGs) were performed according to standard procedures (see online supplement) (18). After a diagnostic PSG and completion of the baseline assessment, participants were randomized to 8 weeks of either active or sham CPAP (see online supplement) (19) and performed a manual CPAP titration PSG or sham CPAP PSG (19). The sham CPAP looked identical to active CPAP, but delivered less than $1.0 \mathrm{~cm} \mathrm{H}_{2} \mathrm{O}$ of pressure (19). All PSGs were scored at a centralized reading laboratory that selected the optimal setting for active treatment. An unmasked polysomnographic technologist performed the CPAP set-ups (Philips Respironics, Monroeville, PA) and distributed CPAP data cards (Philips Respironics Encore SmartCard). Participants sent these cards weekly to the clinical center.

Participants completed the assessment battery at baseline and 8 weeks of intervention. In addition, they completed the FOSQ weekly at home and recorded on the CPAP unit's data card. When the 8-week intervention was completed, participants were informed of their assigned intervention. Those assigned to active treatment were dismissed from the study; those assigned to sham CPAP were crossed over to the active CPAP treatment protocol.

\section{Statistical Analysis}

Sample size was designed to achieve at least $80 \%$ power, using $n=123$ per group with an effect size of at least 0.36 (9). The primary comparison was a modified intent-to-treat (ITT) analysis of participants initiated on the assigned intervention and having a follow-up FOSQ score. The between-group hypotheses for all endpoints were tested using an analysis of covariance model controlling for baseline value, clinical center, and statistically different clinical and demographic characteristics (Table 1). Last Observation Carried Forward (LOCF) imputation was specified for participants missing Week 8 results (for weekly FOSQ Total scores; see Table E1 in online supplement). Statistical significance $(P<0.05)$ of improvements was assessed using paired $t$ tests.

Baseline values for the crossover phase of the study were defined as the results obtained at the completion of the sham CPAP intervention. Paired $t$ tests were performed on results from baseline and end of the 8 -week, active intervention.

\section{RESULTS}

Data collection commenced in 2003 and ended in 2008. Of the 385 participants screened and consented, 281 were randomized (Figure 1). Of these, 42 withdrew after randomization but before exposure to active or sham CPAP (active treatment $n=20$ ) primarily because of time constraints or desiring immediate treatment. These participants were excluded from all analyses. Of the 239 randomized and exposed participants, mean age was $49.5 \pm 10.9$ years in the active CPAP group $(\mathrm{n}=121)$ and $51.7 \pm 11.9$ years in the sham CPAP group $(n=118)$, with $55 \%$ and $63 \%$ males and $79.3 \%$ and $76.3 \%$ whites, respectively (Table 1). The mean ESS score was $15.2 \pm 3.4$ and $14.7 \pm 3.1$ for active and placebo treatment, respectively. Among the 239 randomized and exposed participants, 17 were missing baseline or final FOSQ Total score after applying LOCF, leaving 223 participants in the modified ITT cohort (113 active CPAP, 110 sham CPAP). The only differences at baseline between the two groups were difference in the SF-36 Mental Component and POMS Total Mood Disturbance scores (Table 1). It is unclear why there were differences in mood between the two randomized groups. However, these differences were not clinically meaningful (effect size of -0.29). Regarding lifetime and current medical conditions, the two groups differed with regard to having the lifetime diagnosis of syncope, but there were no statistically significant differences for current conditions (see Tables E3A and E3B). Concomitant medications for both groups are listed in Table E4.

The mean AHI (with $>3 \%$ desaturation) on diagnostic PSG in participants in the active and sham CPAP arms was $12.8 \pm$ 6.4 and $12.5 \pm 6.5$ events per hour, respectively $(P=0.69)$. Sixty-two percent (75 of 121) of participants in the active arm and $64 \%$ (75 of 118) of participants in the sham CPAP arm had mild sleep apnea ( $5 \leqslant$ AHI $<15$ w/3\% desaturation) on baseline testing. On the PSG performed with sham CPAP in those participants randomized to that intervention, the mean AHI 
TABLE 1. PARTICIPANT CHARACTERISTICS AT BASELINE RANDOMIZED AND EXPOSED

\begin{tabular}{|c|c|c|c|c|}
\hline Variable (Mean or \%) & $\begin{array}{l}\text { Participants Randomized to } \\
\text { Active CPAP }(n=121)\end{array}$ & $\begin{array}{l}\text { Participants Randomized to Sham } \\
\text { CPAP }(n=118)\end{array}$ & $P$ Value $^{*}$ & Effect Size \\
\hline Age, yr & $49.5 \pm 10.9$ & $51.7 \pm 11.9$ & 0.13 & -0.54 \\
\hline Percent males & 54.5 & 62.7 & $0.20^{\dagger}$ & N/A \\
\hline Percent African Americans & 15.7 & 16.9 & $0.80^{\dagger}$ & N/A \\
\hline Body mass index, $\mathrm{kg} / \mathrm{m}^{2}$ & $33.2 \pm 6.3$ & $34.2 \pm 7.8$ & 0.42 & -0.14 \\
\hline Weight, lb & $212.9 \pm 44.3$ & $223.5 \pm 22.2$ & 0.32 & -0.30 \\
\hline Apnea-hypopnea index (events/hr w/dsats $>3 \%$ ) & $12.8 \pm 6.4$ & $12.5 \pm 6.5$ & 0.69 & 0.05 \\
\hline Arousal index, events/hr & $33.2 \pm 14$ & $30.4 \pm 11.8$ & 0.09 & 0.22 \\
\hline $\mathrm{O}_{2}$ desaturation index, events $/ \mathrm{hr}$ & $14.3 \pm 6.8$ & $13.9 \pm 6.8$ & 0.67 & 0.06 \\
\hline FOSQ Total score & $13.91 \pm 3.0$ & $14.41 \pm 2.8$ & 0.18 & -0.17 \\
\hline General productivity & $2.90 \pm 0.7$ & $3.01 \pm 0.6$ & 0.21 & -0.17 \\
\hline Vigilance & $2.5 \pm 0.7$ & $2.62 \pm 0.6$ & 0.07 & -0.18 \\
\hline Social outcome & $3.09 \pm 0.7$ & $3.02 \pm 0.8$ & 0.48 & 0.11 \\
\hline Activity level & $2.58 \pm 0.7$ & $2.73 \pm 0.7$ & 0.09 & -0.23 \\
\hline Intimacy and sexual relationships & $2.83 \pm 1.0$ & $3.05 \pm 0.9$ & 0.11 & -0.23 \\
\hline \multicolumn{5}{|l|}{ SF-36 score } \\
\hline Physical activity component & $41.81 \pm 10.8$ & $42.26 \pm 10.2$ & 0.76 & -0.04 \\
\hline Mental health component & $42.92 \pm 11.06$ & $46.04 \pm 10.4$ & 0.04 & -0.29 \\
\hline Epworth total score & $15.21 \pm 3.37$ & $14.66 \pm 3.05$ & 0.20 & 0.17 \\
\hline PVT transformed lapses & $18.49 \pm 29.59$ & $12.94 \pm 21.21$ & 0.12 & 0.19 \\
\hline POMS Total Mood Disturbance & $25.7 \pm 26.3$ & $17.9 \pm 27.5$ & 0.03 & 0.29 \\
\hline Mean arterial blood pressure & $92.5 \pm 8.2$ & $91.6 \pm 8.8$ & 0.46 & 0.11 \\
\hline Systolic blood pressure, day & $124.5 \pm 13.7$ & $124.4 \pm 10.9$ & 0.94 & 0.00 \\
\hline Diastolic blood pressure, day & $76.2 \pm 10.1$ & $74.8 \pm 9.6$ & 0.36 & 0.14 \\
\hline
\end{tabular}

Definition of abbreviations: CPAP $=$ continuous positive airway pressure; FOSQ $=$ Functional Outcomes of Sleep Questionnaire; MAP Index $=$ Multivariable Apnea Prediction Index; POMS = Profile of Mood States; PVT = Psychomotor Vigilance Task; SF-36 = Short Form 36.

${ }^{*} t$ tests for differences.

${ }^{\dagger}$ Fisher exact test.

(with $>3 \%$ desaturation) was $14.6 \pm 12.3$ events per hour and was significantly different from the AHI (with $>3 \%$ desaturation) on the diagnostic study $(P=0.03)$, but the 2.4 event per hour difference was not clinically meaningful (effect size $=0.22$ ). As expected, there was a statistically significant difference between the change with titration in the active CPAP group compared with the sham group $(-11.9,-2.4, P=0.000$, effect

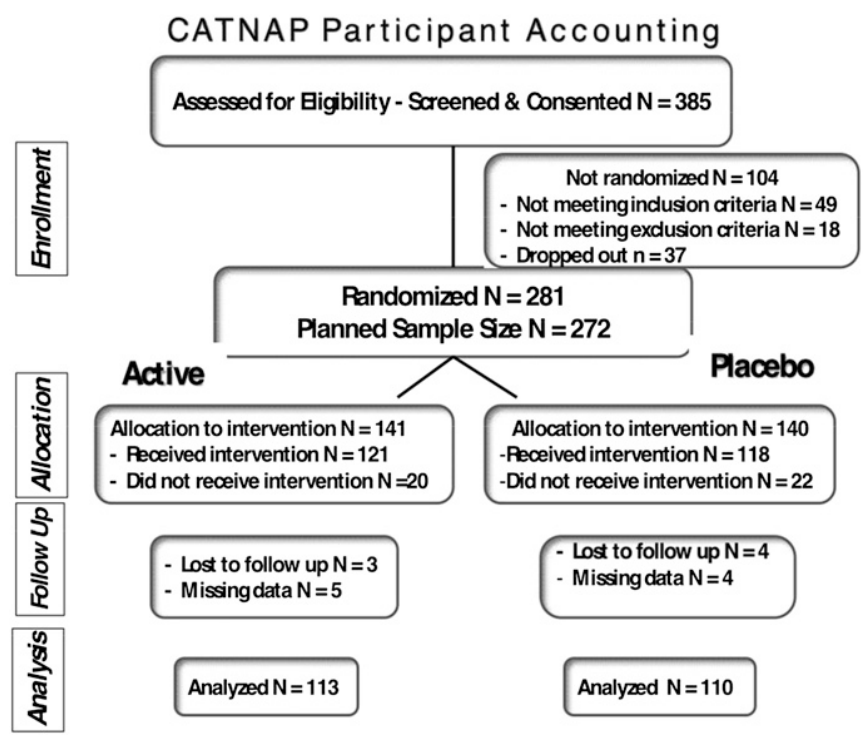

Figure 1. Flowchart of the study profile. Of the 385 participants screened and consented, 281 were randomized and 42 withdrew before any exposure to active or sham continuous positive airway pressure (CPAP). These unexposed participants, although randomized, were excluded from all analyses. There were 239 randomized and exposed participants $(n=121$ active and $n=118$ sham CPAP). CATNAP $=$ CPAP Apnea Trial North American Program. size $=-1.61)$. The active CPAP setting in participants randomized initially to active treatment was $8.1 \pm 2.2$ (range, $4-14$ ) $\mathrm{cm}$ $\mathrm{H}_{2} \mathrm{O}$. On the CPAP titration PSG performed in participants randomized to active CPAP, the mean AHI with greater than $3 \%$ desaturation at the pressure setting selected for subsequent treatment was $0.9 \pm 1.3$ events per hour and was significantly less than that on the diagnostic study $(P<0.0001)$.

\section{Primary Efficacy Analyses}

The mean \pm SD FOSQ Total score at baseline in the primary efficacy cohort was $13.91 \pm 3.02$ and $14.43 \pm 2.78$ in the active and sham CPAP groups, respectively $(P=0.18)$ (Figure 2 and Table 2). The unadjusted mean change in FOSQ Total score from baseline to Week 8 in the modified ITT sample was $0.98 \pm$ 2.89 for the active CPAP group and $-0.14 \pm 2.61$ for the placebo group. Based on the primary (site-weighted and baselineadjusted) analysis of covariance model, the group difference in mean changes in FOSQ Total score from baseline to Week 8 was 0.95 (SE $0.34 ; P=0.006 ; 95 \%$ confidence interval [CI], $0.27-1.62$ ) (Table 3 ). The group difference in mean change corresponded to an effect size of 0.41 (95\% CI, 0.14-0.67).

In descriptive sensitivity analyses, the magnitude of group differences in changes from baseline FOSQ Total score in the modified ITT analysis cohorts was also compared after disabling the LOCF imputation and in per protocol cohorts requiring a mean CPAP use greater than or equal to 4 hours per day (Table 4). These analyses suggested that our primary results may have been conservatively estimated. Seventeen participants required LOCF imputation to be included in the modified ITT analyses. Disabling the LOCF imputation resulted in a more than a $25 \%$ increase in the median percentage improvement in the FOSQ Total score in the active CPAP group, from $5.8 \%$ to $7.3 \%$, but had little effect on the median change in the sham CPAP group. Overall, disabling LOCF increased the (unadjusted) effect size from 0.41 to 0.48 (95\% CI, $0.21-0.76$ ). In the per protocol cohort of active treatment participants with an average daily CPAP use 


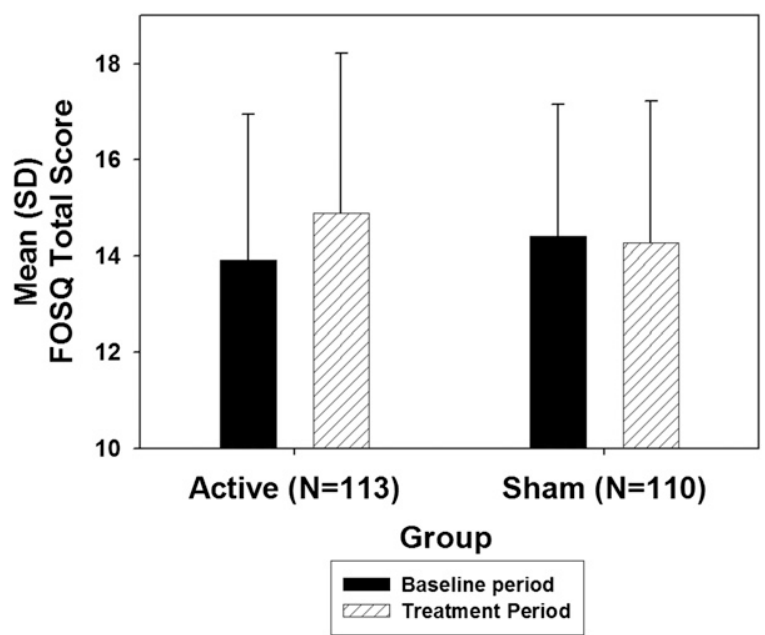

Figure 2. The mean (SD) Functional Outcomes of Sleep Questionnaire (FOSQ) Total score by treatment group in the primary efficacy cohort before and after the 8-week treatment period.

of at least 4 hours, the median percentage change in FOSQ Total score increased another $56 \%$, from $7.3 \%$ to $11.4 \%$. The median per protocol change in the sham CPAP group was $-1.2 \%$ to $1.9 \%$. There was little change in the effect size for this cohort (effect size $=0.49 ; 95 \%$ CI, 0.07-0.90).

\section{Secondary Efficacy Analyses}

The adjusted mean differences between groups showed significant improvements in all FOSQ subscale scores except Social Outcome, and Intimacy and Sexual Relationships (Table 3). The adjusted mean changes from baseline to Week 8 for the other secondary outcome measures are in Tables E6 and E7. Significant improvements in the active CPAP group compared with the sham CPAP group occurred in the following SF-36 subscales: Physical Component, Physical Functioning, Bodily Pain, General Health, and Vitality $(P<0.04)$. The unadjusted mean change in the ESS score was $-2.6 \pm 4.3$ for the active group $(P<0.00001)$ and $-0.5 \pm 3.5$ in the sham group $(P=$ $0.12)$. The adjusted mean difference between groups was -1.8 (SE, 0.5) $(P=0.001 ; 95 \%$ CI, bounds -2.8 to -0.8$)$. Total Mood Disturbance on the POMS and the subscales of Fatigue, Confusion-Bewilderment, and Vigor were significantly improved in the active versus sham CPAP group $(P \leqslant 0.014)$. No significant difference was observed in the change of the number of lapses on PVT between the two groups $(P=0.12)$.

The 48-hour ambulatory blood pressure recordings were analyzed for mean adjusted change in daytime pressure, nocturnal pressure, and nocturnal dipping of the systolic, diastolic, and mean arterial pressures and heart rate. The study was not powered for these secondary outcomes and, because of technical difficulties, results for the modified ITT analysis were obtained in only about half of the participants in each group. The sole significant difference in blood pressure between the two groups was the mean adjusted change in daytime diastolic blood pressure $(P=0.048)$.

\section{CPAP Use}

The mean \pm SD duration of CPAP use was $4.0 \pm 2.0$ and $3.1 \pm$ 2.1 hours per day in the active CPAP and sham CPAP groups, respectively $(t[313])=3.3 ; P=0.001)$. We conducted Pearson correlations to determine the strength of the linear association between mean daily hours of CPAP use and change in FOSQ Total score. The correlation in the active treatment group was moderately large and statistically significant $(r=0.25 ; P=$ $0.008 ; \mathrm{n}=101)$. In contrast, the correlation in the sham CPAP group was small and not statistically significant $(r=0.15 ; P=$ $0.12 ; \mathrm{n}=97)$. Thus, $6.4 \%$ of the variance in FOSQ Total score improvements could be explained by a linear association with mean CPAP use in the active treatment group. In contrast, only $2.3 \%$ of FOSQ Total score improvement variance was explained in the sham CPAP group, and the association did not achieve statistical significance.

\section{Crossover Cohort Analyses}

Of the 118 subjects randomized and exposed to sham CPAP, 102 (86.4\%) were enrolled into the 8-week active CPAP intervention. Of these 99 had a FOSQ Total score at the end of their sham CPAP intervention (i.e., the baseline measurement used in the crossover analysis). Their demographic characteristics are reported in Table E8. The mean (SD) improvement in FOSQ Total score from the beginning to the end of the crossover phase $(\mathrm{n}=$ 91) was $1.73 \pm 2.50(t[90]=6.59 ; P<0.00001)($ see Table E5) with a moderately large standardized effect size of 0.69 . Statistically robust improvements in function were observed for all FOSQ subscale domains. Although the standard effect sizes varied, all were at least moderately large (see Table E5). Significant improvements in the crossover cohort were also observed in ESS score with a change of $2.3 \pm 4.0(P<0.001)$; all component scores of the SF-36 $(P<0.020)$; and several domains of the POMS (Fatigue, Confusion-Bewilderment, Vigor, and Total Mood Disturbance; $P<0.003)$. The mean change in the number of PVT lapses was $-3.93 \pm 13.46$ (SD) $(P=0.011)$. No significant changes in blood pressure measures after 8-weeks of active treatment were observed in the crossover cohort.

TABLE 2. THE UNADJUSTED MEAN CHANGES IN FOSQ TOTAL AND COMPONENT SCORES AFTER THE 8-WEEK INTERVENTION WITH ACTIVE VERSUS SHAM CPAP

\begin{tabular}{lccccc}
\hline & \multicolumn{2}{c}{ Active CPAP Group $(n=113)$} & & \multicolumn{2}{c}{ Sham CPAP Group $(n=110)$} \\
\cline { 2 - 3 } \cline { 5 - 6 } Variable & Mean Change & $P$ Value* & & Mean Change \pm SD & $P$ Value $^{*}$ \\
\hline FOSQ Total score & $0.98 \pm 2.89$ & 0.0005 & & $-0.14 \pm 2.61$ & 0.57 \\
General productivity & $0.20 \pm 0.62$ & 0.0007 & & $0.00 \pm 0.61$ & 0.97 \\
Vigilance & $0.16 \pm 0.77$ & 0.03 & & $-0.12 \pm 0.81$ & 0.14 \\
Social outcome & $0.08 \pm 0.83$ & 0.34 & & $-0.02 \pm 0.78$ & 0.86 \\
Activity level & $0.26 \pm 0.70$ & 0.0001 & & $-0.05 \pm 0.56$ & 0.32 \\
Intimacy and sexual relationships & $0.09 \pm 1.11$ & 0.42 & & $-0.14 \pm 1.06$ & 0.22 \\
\hline
\end{tabular}

Definition of abbreviations: CPAP = continuous positive airway pressure; FOSQ = Functional Outcomes of Sleep Questionnaire.

* Paired $t$ tests. 
TABLE 3. CHANGES FROM PRETREATMENT BASELINE TO THE FINAL TREATMENT PERIOD IN THE ITT SAMPLE (PRIMARY AND SUPPORTING TESTS FOR EFFICACY)

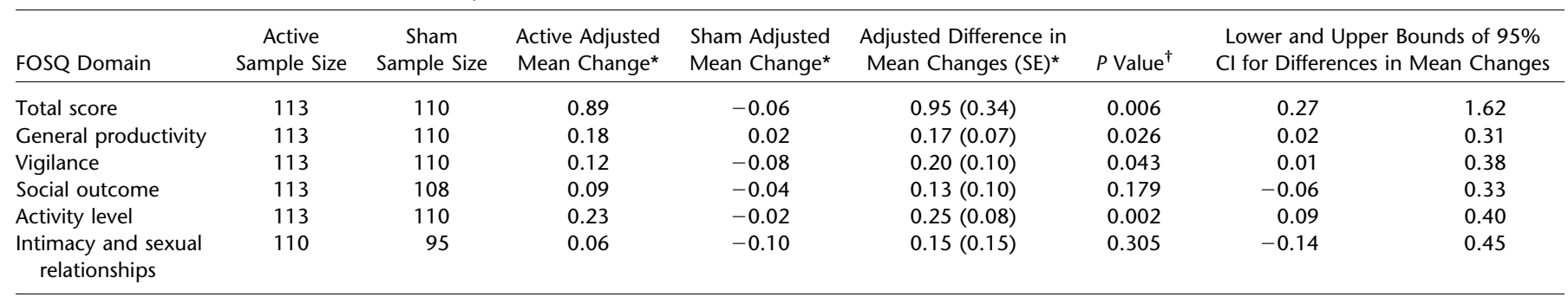

Definition of abbreviations: $\mathrm{Cl}=$ confidence interval; FOSQ = Functional Outcomes of Sleep Questionnaire; ITT = intent to treat.

* Adjusted mean changes and adjusted differences in mean changes were estimated as site-total-sample-size weighted values controlling for treatment group differences in mean pretreatment baseline values. Individual baseline values were used for individual FOSQ component scores.

${ }^{\dagger} P$ value from type II sum of squares estimated by way of analysis of covariance. To produce site weighted comparisons the analysis of covariance model included main effects for treatment group, site, and pretreatment baseline value.

\section{Safety Analysis}

Table E2 summarizes the overall safety experience in the two intervention groups. There were few important adverse events with no significant group differences.

\section{DISCUSSION}

To our knowledge, this multisite, double-blind, RCT presents the findings from the largest placebo-controlled investigation of the efficacy of CPAP treatment in sleepy patients with milder OSA. Sleepy patients with mild and moderately severe OSA had greater functional improvement after 8 weeks of CPAP therapy compared with sham CPAP. The group difference in change in FOSQ Total score, ESS, Physical Component of the SF-36, and Total Mood Disturbance were highly significant and clinically relevant as indicated by the effect size. Of note is that the mean change in FOSQ Total score was quite similar to the difference in this score between CPAP and usual care/placebo in studies that have included a wide spectrum of disease severity (12). As a multisite study conducted at large and smaller clinical practice sites, our results are highly generalizable. Moreover, our sample reflects the typical age associated with OSA and had almost equal representation of sexes. Our protocol was designed to have the least impact on the routine care provided at the clinical centers; thus, we believe that our results have high external validity and are applicable to outcomes associated with the management of patients treated with CPAP at most sleep centers.

A major strength of our study is the use of sham CPAP for the placebo intervention. The few studies that have explored the impact of CPAP treatment in milder OSA have used conservative therapy or placebo tablets as controls $(10,20-23)$. There has been criticism that these controls make it difficult to mask the participants and research personnel and do not provide the participants with the same experience as CPAP (24). In response, we used as our control sham CPAP that does not deliver effective pressure, adversely affect sleep, or reduce AHI (19). Comparison of the PSGs performed at baseline with and without sham CPAP did show changes in some secondary PSG measurements (19). However, the lack of significant change in any functional outcome measure in the sham-CPAP group provides strong evidence that these PSG differences were not of clinical significance. Sham CPAP allowed a true efficacy comparison with active CPAP, especially related to subjective assessments $(24,25)$. Our finding that active CPAP treatment compared with placebo enhanced daily functioning is consistent with previous RCTs conducted primarily in those with moderate to severe OSA (9-12).

Results of recent meta-analyses of CPAP RCTs $(9,10)$ prompted the recommendation to treat moderate to severe OSA as a practice standard (8). However, lacking conclusive evidence in those with more mild disease, the American Academy of Sleep Medicine indicated that CPAP is an optional patient-care strategy for enhancing quality of life in this population (8). The improvement we found in functional status in sleepy patients with milder OSA is consistent with studies of those with more severe disease and supports the application of CPAP therapy as standard in patients with milder OSA who have symptoms of daytime sleepiness (9).

TABLE 4. FOSQ TOTAL SCORE SUMMARY STATISTICS BY TREATMENT GROUP IN THE INTENT-TO-TREAT* AND PER PROTOCOL SAMPLES ${ }^{\dagger}$

\begin{tabular}{|c|c|c|c|c|c|c|c|c|c|c|c|c|c|c|}
\hline \multirow[b]{2}{*}{ Sample } & \multirow[b]{2}{*}{ Treatment } & \multirow[b]{2}{*}{$\mathrm{N}$} & \multicolumn{3}{|c|}{$\begin{array}{l}\text { Pretreatment Baseline } \\
\text { FOSQ Total Score }\end{array}$} & \multicolumn{3}{|c|}{$\begin{array}{l}\text { Final Treatment Period }{ }^{\ddagger} \\
\text { FOSQ Total Score }\end{array}$} & \multicolumn{3}{|c|}{ Change from Baseline } & \multicolumn{3}{|c|}{ Percent Change from Baseline } \\
\hline & & & Mean & SD & Median & Mean & SD & Median & Mean & SD & Median & Mean & SD & Median \\
\hline \multirow[t]{2}{*}{ Intent-to-treat ${ }^{\S}$} & Active & 113 & 13.92 & 3.02 & 14.39 & 14.89 & 3.32 & 15.29 & 0.98 & 2.89 & 0.90 & $9.1 \%$ & $23.3 \%$ & $5.8 \%$ \\
\hline & Sham & 110 & 14.41 & 2.75 & 14.70 & 14.27 & 2.96 & 14.79 & -0.14 & 2.61 & -0.21 & $0.6 \%$ & $19.2 \%$ & $-1.3 \%$ \\
\hline \multirow[t]{2}{*}{ LOCF disabled } & Active & 105 & 13.96 & 2.98 & 14.48 & 15.19 & 3.07 & 15.40 & 1.23 & 2.61 & 1.13 & $11 \%$ & $22.2 \%$ & $7.3 \%$ \\
\hline & Sham & 101 & 14.40 & 2.76 & 14.62 & 14.41 & 2.82 & 14.79 & 0.01 & 2.40 & -0.19 & $1.7 \%$ & $18.3 \%$ & $-1.2 \%$ \\
\hline \multirow[t]{2}{*}{ Per protocol } & Active & 52 & 13.07 & 3.15 & 13.61 & 14.75 & 3.59 & 15.39 & 1.68 & 2.88 & 1.49 & $15.3 \%$ & $26.2 \%$ & $11.4 \%$ \\
\hline & Sham & 41 & 13.93 & 2.66 & 13.95 & 14.25 & 2.86 & 14.54 & 0.32 & 2.66 & 0.25 & $4.0 \%$ & $19.7 \%$ & $1.9 \%$ \\
\hline
\end{tabular}

Definition of abbreviations: CPAP = continuous positive airway pressure; FOSQ = Functional Outcomes of Sleep Questionnaire; LOCF = Last Observation Carried Forward.

* The Intent-to-Treat sample includes all randomized patients exposed to active CPAP or sham CPAP treatment during the post-randomization treatment period.

${ }^{\dagger}$ The Per Protocol sample includes randomization patients meeting criterion for inclusion in the Intent-to-Treat sample who also meet CPAP compliance criterion and

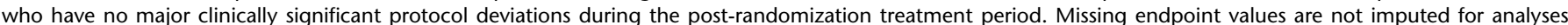
involving the Per Protocol sample.

${ }^{\ddagger}$ Final treatment period FOSQ endpoints are defined at Week 8 or last available among Weeks 1-7 based on available smartcard data.

$\S$ Primary efficacy analyses were performed in the Intent-to-Treat sample.

" Secondary efficacy analyses were performed in the Per Protocol sample. 
As the primary manifestation of OSA, daytime sleepiness has been the most common treatment outcome investigated. In a metaanalysis of seven RCTs of the impact of CPAP on selfrated sleepiness in mild sleep apnea, Marshall and colleagues (26) reported that ESS scores were significantly improved after CPAP treatment by 1.2 points $(95 \% \mathrm{CI}, 0.5-1.9 ; P=0.001)$, after controlling for placebo effects. These findings are consistent with our results showing an adjusted difference in mean change between the treatment arms of $-1.8(95 \% \mathrm{CI},-0.75$ to $-2.82 ; P=$ $0.001)$ indicating that participants perceived greater alertness with CPAP treatment. We believe that our larger sample size and lower average dropout rate compared with the studies included in the metaanalysis accounts for our more robust findings.

\section{Study Limitations}

A concern was the mean duration of daily CPAP treatment. Despite a protocol to promote CPAP use through pretreatment education followed by weekly contact that included troubleshooting and motivation (see online supplement), our mean daily CPAP use was only $4.0 \pm 2.0$ and $3.1 \pm 2.1$ hours per day in the active CPAP and sham CPAP groups, respectively. We did not achieve the desired 6 hours or greater nightly use, nor did we get equal exposure to intervention between the two groups. Previous RCTs also report mean use of less than 5 hours $(9,11)$. Despite the statistically significant improvement in daytime sleepiness, at the end of the treatment period $71 \%$ of the active treatment arm had an ESS total score large than the normal value of 10 (13). Eightythree percent of the sham group self-reported daytime sleepiness. The improvement in the active group relative to sham was statistically significant (chi-square, $P=0.03$ ). The persistence of daytime sleepiness on treatment is not novel to this study and has been previously reported (27-29). It is speculated that the residual sleepiness evident in our study may be related to the less than optimal nightly duration of CPAP use of $4.0 \pm 2.0$ hours rather than the desired greater than 6 hours of use (29). The lower mean daily adherence to sham CPAP than active CPAP in our study was likely associated with the perception of decreased benefit. Because the duration of treatment use in our study is similar to the 4-hour average in the clinical setting (9), expectations for clinical outcomes for milder OSA would be consistent with our findings. Moreover, although we showed that the FOSQ Total score improves linearly with increasing hours of use (i.e., more is better), some benefit was achieved even with relatively low usage time (29).

\section{Conclusions}

This multisite, double-blind RCT is the first placebo-controlled study using sham CPAP in sleepy patients with mild to moderate OSA and demonstrates improved quality of life and symptom reduction with CPAP treatment. It remains unclear whether those with milder OSA who do not report daytime sleepiness would experience similar benefits. Given the high prevalence of OSA in the general public, this study importantly suggests significant value in treating sleepy patients with mild to moderate disease. Although other forms of treatment are available, such as dental appliances, CPAP is the primary treatment for OSA. Our results demonstrate that CPAP therapy for sleepy patients with milder OSA can confer significant health benefits.

$\underline{\text { Author disclosures }}$ are available with the text of this article at www.atsjournals.org.

Acknowledgment: The following individuals contributed to the study: Mary JonesParker, RPSGT, and Haideliza Soto-Calderon (CATNAP PSG Reading Center, University of Pennsylvania) and Shawn Ballard and Bridget Small (Clinical Research Computing Unit of the Center for Clinical Epidemiology and Biostatistics, University of Pennsylvania). Research coordinators and PSG technologists at the clinica centers included Mala Ramu, Leila MacBean, and Nina Marinkovic, University of Western Ontario; Kara Barrett, Roslyn Seitz, and Jasmine Konn, Emory University; Bien Pagan-Lee and Ming Chen, New York University; Kristen Cruz, Asha John, Ana Hallinan, and Rod Massop, North Shore-Long Island Jewish Health System; and Jen Goldschmeid and Allison Rankin, National Jewish Health. Dr. David Dinges, University of Pennsylvania, contributed to data quality control for the Psychomotor Vigilance Task measurements. The members of the Data Safety Monitoring Board were Dr. John Fleetham (Chair), University of British Columbia; Dr. Susan Ellenberg, University of Pennsylvania; Dr. Vern Chinchilli, Penn State University; Rochelle Goldberg, M.D., REM Medical; and Wolfgang SchmidtNowara, M.D., Sleep Medicine Associates of Texas. Kevin Granfield provided editorial assistance.

\section{References}

1. Young T, Peppard PE, Gottlieb DJ. Epidemiology of obstructive sleep apnea: a population health perspective. Am J Respir Crit Care Med 2002;165:1217-1239.

2. Gottlieb DJ, Whitney CW, Bonekat WH, Iber C, James GD, Lebowitz M, Nieto FJ, Rosenberg CE. Relation of sleepiness to respiratory disturbance index: the Sleep Heart Health study. Am J Respir Crit Care Med 1999;159:502-507.

3. Sleep-related breathing disorders in adults: recommendations for syndrome definition and measurement techniques in clinical research. The report of an American Academy of Sleep Medicine task force. Sleep 1999;22:667-689.

4. Selim B, Won C, Yaggi HK. Cardiovascular consequences of sleep apnea. Clin Chest Med 2010;31:203-220.

5. Ellen RL, Marshall SC, Palayew M, Molnar FJ, Wilson KG, Man-SonHing M. Systematic review of motor vehicle crash risk in persons with sleep apnea. J Clin Sleep Med 2006;2:193-200.

6. Ulfberg J, Carter N, Talback M, Edling C. Excessive daytime sleepiness at work and subjective work performance in the general population and among heavy snorers and patients with obstructive sleep apnea. Chest 1996;110:659-663.

7. Lui MM, Ip MS. Disorders of glucose metabolism in sleep-disordered breathing. Clin Chest Med 2010;31:271-285.

8. Kushida CA, Littner MR, Hirshkowitz M, Morgenthaler TI, Alessi CA, Bailey D, Boehlecke B, Brown TM, Coleman J Jr, Friedman L, et al. Practice parameters for the use of continuous and bilevel positive airway pressure devices to treat adult patients with sleep-related breathing disorders. Sleep 2006;29:375-380.

9. Gay P, Weaver T, Loube D, Iber C. Evaluation of positive airway pressure treatment for sleep related breathing disorders in adults. Sleep 2006;29:381-401.

10. Giles TL, Lasserson TJ, Smith BH, White J, Wright J, Cates CJ. Continuous positive airways pressure for obstructive sleep apnoea in adults. Cochrane Database Syst Rev 2006;3:CD001106.

11. Sharma SK, Agrawal S, Damodaran D, Sreenivas V, Kadhiravan T, Lakshmy R, Jagia P, Kumar A. CPAP for the metabolic syndrome in patients with obstructive sleep apnea. N Engl J Med 2011;365: 2277-2286.

12. McDaid C, Griffin S, Weatherly H, Duree K, van der Burgt M, van Hout S, Akers J, Davies RJ, Sculpher M, Westwood M. Continuous positive airway pressure devices for the treatment of obstructive sleep apnoeahypopnoea syndrome: a systematic review and economic analysis. Health Technol Assess 2009;13:iii-iv, xi-xiv, 1-119, 143-274.

13. Johns MW. Sensitivity and specificity of the multiple sleep latency test (MSLT), the maintenance of wakefulness test and the Epworth Sleepiness Scale: failure of the MSLT as a gold standard. J Sleep Res 2000;9:5-11.

14. Weaver TE, Laizner AM, Evans LK, Maislin G, Chugh DK, Lyon K, Smith PL, Schwartz AR, Redline S, Pack AI, et al. An instrument to measure functional status outcomes for disorders of excessive sleepiness. Sleep 1997;20:835-843.

15. Ware JE, Snow KK, Kosinski M, Gandek B. Sf 36 health survey. Manual and interpretation guide. Boston: The Health Institute, New England Medical Center; 1990.

16. Dinges D, Powell J. Microcomputer analyses of performance on a portable, simple visual RT task during sustained operations. Behav Res Methods Instrum Comput 1985;17:652-655.

17. McNair DM, Lorr M, Druppleman LF. EITS manual for the profile of mood states. San Diego: Educational and Industrial Test Services; 1971. 
18. Kushida CA, Littner MR, Morgenthaler T, Alessi CA, Bailey D, Coleman J, Friedman L, Hirshkowitz M, Kapen S, Kramer M, et al. Practice parameters for the indications for polysomnography and related procedures: an update for 2005. Sleep 2005;28:499-521.

19. Rodway GW, Weaver TE, Mancini C, Cater J, Maislin G, Staley B, Ferguson KA, George CF, Schulman DA, Greenberg H, et al. Evaluation of sham-CPAP as a placebo in CPAP intervention studies. Sleep 2010;33:260-266.

20. Barnes M, Houston D, Worsnop CJ, Neill AM, Mykytyn IJ, Kay A, Trinder J, Saunders NA, Douglas McEvoy R, et al. A randomized controlled trial of continuous positive airway pressure in mild obstructive sleep apnea. Am J Respir Crit Care Med 2002;165:773-780.

21. Barnes M, McEvoy RD, Banks S, Tarquinio N, Murray CG, Vowles N, Pierce RJ. Efficacy of positive airway pressure and oral appliance in mild to moderate obstructive sleep apnea. Am J Respir Crit Care Med 2004;170:656-664.

22. Engleman HM, Martin SE, Deary IJ, Douglas NJ. Effect of CPAP therapy on daytime function in patients with mild sleep apnoea/hypopnoea syndrome. Thorax 1997;52:114-119.

23. Engleman HM, Kingshott RN, Wraith PK, Mackay TW, Deary IJ, Douglas NJ. Randomized placebo-controlled crossover trial of continuous positive airway pressure for mild sleep apnea/hypopnea syndrome. Am J Respir Crit Care Med 1999;159:461-467.
24. Wright J, Johns R, Watt I, Melville A, Sheldon T. Health effects of obstructive sleep apnoea and the effectiveness of continuous positive airways pressure: a systematic review of the research evidence. BMJ 1997:314:851-860.

25. Jenkinson C, Davies RJO, Mullins R, Stradling JR. Comparison of therapeutic and subtherapeutic nasal continuous positive airway pressure for obstructive sleep apnoea: a randomised prospective parallel trial. Lancet 1999;353:2100-2105.

26. Marshall NS, Barnes M, Travier N, Campbell AJ, Pierce RJ, McEvoy RD, Neill AM, Gander PH. Continuous positive airway pressure reduces daytime sleepiness in mild to moderate obstructive sleep apnoea: a meta-analysis. Thorax 2006;61:430-434.

27. Schwartz JR, Hirshkowitz M, Erman MK, Schmidt-Nowara W. Modafinil as adjunct therapy for daytime sleepiness in obstructive sleep apnea: a 12-week, open-label study. Chest 2003;124:2192-2199.

28. Bittencourt LR, Lucchesi LM, Rueda AD, Garbuio SA, Palombini LO, Guilleminault C, Tufik S. Placebo and modafinil effect on sleepiness in obstructive sleep apnea. Prog Neuropsychopharmacol Biol Psychiatry 2008;32:552-559.

29. Weaver TE, Maislin G, Dinges DF, Bloxham T, George CF, Greenberg H, Kader G, Mahowald M, Younger J, Pack AI. Relationship between hours of CPAP use and achieving normal levels of sleepiness and daily functioning. Sleep 2007;30:711-719. 